\title{
Predictive, Reactive and Replication-based Load Balancing of Tasks in Chameleon and sam(oa) ${ }^{2}$
}

\author{
Philipp Samfass \\ Technical University of Munich \\ Department of Informatics \\ Garching, Germany \\ samfass@in.tum.de \\ Minh Thanh Chung \\ Ludwig-Maximilians-Universitaet \\ MNM-Team \\ Munich, Germany \\ minh.thanh.chung@ifi.lmu.de
}

\author{
Jannis Klinkenberg \\ RWTH Aachen University \\ IT Center \\ Aachen, Germany \\ j.klinkenberg@itc.rwth-aachen.de \\ Michael Bader \\ Technical University of Munich \\ Department of Informatics \\ Garching, Germany \\ bader@in.tum.de
}

\begin{abstract}
Increasingly complex hardware architectures as well as numerical algorithms make balancing load in parallel numerical software for adaptive mesh refinement an inherently difficult task, especially if variability of system components and unpredictability of execution time comes into play. Yet, traditional predictive load balancing strategies are largely based on cost models that aim to predict the execution time of computational tasks. To address this fundamental weakness, we present a novel reactive load balancing approach in distributed memory for MPI+OpenMP parallel applications that is based on keeping tasks speculatively replicated on multiple MPI processes. Replicated tasks are scheduled fully reactively without the need of a predictive cost model. Task cancellation mechanisms help to keep the overhead of replication minimal by avoiding redundant computation of replicated tasks. We implemented our approach in the Chameleon library for reactive load balancing building upon previous work on reactive task migration. Our experiments in the parallel dynamic adaptive mesh refinement software $\operatorname{sam}(\mathrm{oa})^{2}$ demonstrate performance improvements in the presence of wrong cost models and artificially introduced noise to simulate imbalances coming from hardware variability.
\end{abstract}

\section{CCS CONCEPTS}

\section{- Computing methodologies $\rightarrow$ Parallel algorithms.}

\section{KEYWORDS}

load balancing, MPI, OpenMP, reactive, replication, overdecomposition, tasking

\section{ACM Reference Format:}

Philipp Samfass, Jannis Klinkenberg, Minh Thanh Chung, and Michael Bader. 2021. Predictive, Reactive and Replication-based Load Balancing of Tasks in Chameleon and sam(oa $)^{2}$. In Platform for Advanced Scientific

Permission to make digital or hard copies of part or all of this work for personal or classroom use is granted without fee provided that copies are not made or distributed for profit or commercial advantage and that copies bear this notice and the full citation on the first page. Copyrights for third-party components of this work must be honored.

For all other uses, contact the owner/author(s)

PASC '21, July 5-9, 2021, Geneva, Switzerland

(c) 2021 Copyright held by the owner/author(s)

ACM ISBN 978-1-4503-8563-3/21/07.

https://doi.org/10.1145/3468267.3470574
Computing Conference (PASC '21), fuly 5-9, 2021, Geneva, Switzerland. ACM, New York, NY, USA, 10 pages. https://doi.org/10.1145/3468267.3470574

\section{INTRODUCTION}

Balancing load in parallel numerical simulations becomes increasingly challenging. On the software side, this is driven by modern complex numerics: for instance, the ADER-DG numerical scheme with a-posteriori limiting [28] dynamically adds computational work if an element-local solution candidate is detected to be physically invalid, and it solves non-linear element-local systems of equations taking an unpredictable number of iterations [21]. Dynamically adaptive mesh refinement (AMR), where the grid and computational load changes during the simulation, adds even further complexity (see Sec. 2 on related work). On the hardware side, techniques such as dynamic voltage and frequency scaling render the common assumption of "equal work implies equal execution time" increasingly inaccurate [2,6].

Applications need to adopt new load balancing strategies to deal with unpredictability at run time. This problem is particularly hard to solve in distributed memory, where the cost and overhead of fine-granular load re-distribution typically exceeds its benefit. In previous work, Chameleon, a library for reactive load balancing of MPI+OpenMP task-parallel applications, was presented [16]. Chameleon extends MPI+OpenMP applications by fine-granular distributed tasking, i.e., tasks can be migrated to other ranks and be executed there. It implements mechanisms for fully overlapping the communication overhead, continuously monitoring of the current processor load and for making appropriate migration decisions. It was demonstrated that Chameleon provides a second useful load balancing dimension that works alongside of traditional predictionbased methods such as domain decomposition [23].

In this paper, we build on this work introducing and evaluating a novel reactive load balancing approach in Chameleon that is based on replication of tasks. Replication of work to minimize time to solution is often used in the cloud computing context (e.g., speculative execution in MapReduce [8]), where jobs are replicated onto multiple nodes and computed in parallel. The first node to finish delivers the job result and the work on other nodes may be canceled. This idea has not been explored much in HPC, though. Our 
approach speculatively keeps tasks replicated on multiple ranks. Through task cancellation, it mostly avoids expensive duplicate computations - an important property in capability computing. Task replication paired with cancellation and reactive scheduling allows us to quickly react to dynamically arising load imbalances that cannot be predicted at run time. It also increases robustness in the presence of wrong predictive load balancing decisions.

Our main contributions are as follows:

- We define reactive and predictive task-based load balancing, discussing their differences and weaknesses.

- We present the design of a novel approach for task-based reactive load balancing that uses speculative, fully overlapping replication of tasks and task cancellation. The approach does not rely on any predictions.

- We discuss the implementation of task-based replication in Chameleon and evaluate its reactive load balancing techniques in the $\operatorname{sam}(\mathrm{oa})^{2}$ framework [17] for parallel AMR with respect to their potential for reacting to unpredictable load imbalances.

While we focus on the concrete AMR solver sam $(\mathrm{oa})^{2}$, we point out that the Chameleon library is designed for arbitrary MPI+OpenMP task-parallel applications and that the discussion should therefore be generalizable to applications that require dynamic load balancing.

\section{RELATED WORK}

Load balancing for simulations that require parallel AMR typically relies on domain decomposition to evenly distribute the computational work across processes. For tree-structured meshes (as used in this work), well-known examples include the p4est library [5], which implements a forest of octrees as grid data structure that is sequentialized along Morton order. Octants can be repartitioned between MPI processes based on individual weights for each octant. The Peano framework [26] builds an adaptive Cartesian mesh from a spacetree, which is traversed along a Peano space-filling curve. Subtrees of the global space tree are assigned to MPI processes, allowing for a parallel traversal of the mesh. Using the PARAMESH framework for adaptive octree-based meshes, the FLASH code [9] simulates various physical models at multiple scales. Combined with adaptivity, it is particularly challenging to decompose the grid in FLASH such that a good load distribution is achieved: e.g., optimizing load balance for one physical operator may have an adverse effect on the load balance of another physical operator.

Beyond application-specific domain decomposition, there are also more general load balancing approaches such as task-based load balancing. Both OpenMP [18] and Intel TBB [22] provide concepts for a task-based execution model and several implementations of these models facilitate work stealing. However, work stealing in OpenMP and Intel TBB is restricted to a process's address space.

Another method (called LeWi, lend when idle) is implemented in the dynamic load balancing library $D L B$ [13]. DLB intercepts blocking MPI calls and lends CPU cores from an underloaded blocking process to an overloaded process. While this provides load balance between MPI processes, it only works within a compute node, which implies that multiple MPI processes must be started on each compute node.
This work focuses on reactive task-based load balancing between MPI processes which is orthogonal to domain decomposition in established AMR frameworks and rather adds a second level of lightweight load balancing by temporarily migrating tasks to other ranks and executing them there. A prototype of this technique was implemented in $\operatorname{sam}(\mathrm{oa})^{2}$ [23]. This approach was extracted into a separate library (Chameleon) to also make it available to other bulk-synchronous hybrid MPI+OpenMP applications [15, 16]. A modified variant of reactive task-based load balancing was also implemented in the ExaHyPE engine [24]. In ExaHyPE, task replication is exploited in combination with teaMPI [25]: MPI processes are replicated transparently and share tasks between the replicated processes addressing the potential of process replication for fault resilience. In contrast to this previous work, we focus on replication of tasks for load balancing in this work.

\section{TERMINOLOGY}

We start with several definitions to obtain a formalism of task-based load balancing that will help to explain the key ideas we use and to clarify our assumptions.

\subsection{Task-based Load Balancing}

We assume that a parallel program can be decomposed into distinct execution phases for executing tasks in parallel by multiple processes. Each such parallel task execution phase is followed by synchronization (e.g., bulk-synchronous processing of tasks) such as an explicit time step for a numerical PDE solver followed by a maximum reduction over allowed time step sizes.

In each task execution phase, each of $n_{t}$ independent tasks $i \in$ $T=\left\{1, \ldots, n_{t}\right\}$ needs to be executed on one of $n_{p}$ processes $p \in$ $P=\left\{1, \ldots, n_{p}\right\}$. Using over-decomposition (i.e., $n_{t} \gg n_{p}$ ) allows to better balance load. Finally, we assume tasks to be atomic, i.e., a scheduled task will run on a specific core until termination. For each execution phase, tasks are physically distributed across processes before they can be executed. In particular, they must reside in the respective process's memory address space. We call this physical (re-)assignment of tasks including the required data migration a load distribution step.

Each task has an associated execution wall clock time $w_{i} \geq 0$, which depends on various parameters, such as the actual instructions executed by the task and its input parameters. Further, hardware characteristics such as the executing processor, its CPU clock frequency and the available memory bandwidth influence execution time. Therefore, $w_{i}$ can only be measured at run time.

Whenever a load distribution step is executed, a load balancing strategy decides which tasks to assign to which process according to a task assignment:

Definition 3.1 (Task Assignment). A task assignment $M: T \rightarrow$ $P$ establishes a mapping between each ready task $i$ and its designated process $p=M(i)$ to run task $i$.

Let $T_{p, M} \subset T$ denote the set of tasks assigned to process $p$ under the task assignment $M$. We can then define:

Definition 3.2 (Wall Clock Execution Time). The wall clock execution time $W_{p, M}$ on process $p$ under the task assignment $M$ is $W_{p, M}:=\sum_{i \in T_{p, M}} w_{i}$ 


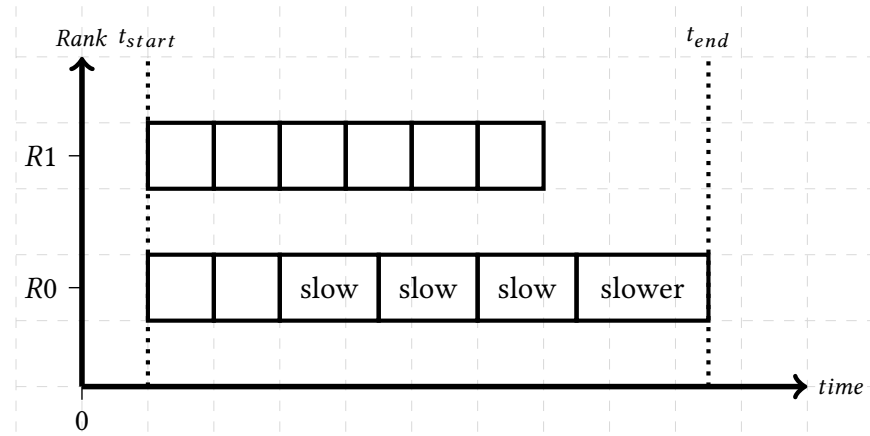

Figure 1: Load imbalance in a parallel task execution phase (dotted vertical lines): Boxes reflect task executions on the time axis; their length indicates elapsed wall clock time. We assume that rank 0 slows down, e.g., due to clock frequency variations, causing load imbalance and idle time at rank 1.

With bulk-synchronous processing of tasks, the process with the longest wall clock execution time determines the wall clock execution time of the task execution phase. We define:

Definition 3.3 (Parallel Wall Clock Execution Time). The parallel wall clock execution time for a task execution phase using a specific task assignment mapping $M$ is $W_{p a r, M}:=\max _{p \in P} W_{p, M}$

This parallel wall clock execution time is observed by the application user. This motivates the following definition:

Definition 3.4 (Bottleneck Process). The process $p_{b}$ that determines the parallel wall clock execution time is called the bottleneck process, $p_{b}:=\arg \max _{p \in P} W_{p, M}$.

In this setting, minimizing the wall clock execution time of $p_{b}$ minimizes the overall parallel execution time. Hence, each load balancing strategy needs to come up with a "good" task assignment.

\subsection{Predictive Load Balancing}

As the task execution time $w_{i}$ cannot be known exactly a-priori, most load balancing algorithms rely on a runtime model $f: \mathbb{R}^{n} \rightarrow$ $R$ for the predicted execution time $\hat{w}_{i}=f\left(c_{i}\right)$ of a task $i$. Here, $c_{i} \in \mathbb{R}^{n}$ is a vector of cost indicators for task $i$, e.g., the number of flops or some other other measure of work. This gives rise to the following definition:

Definition 3.5 (Predictive Load Balancing). Predictive load balancing implicitly or explicitly relies on a runtime model $f$ for the execution time of tasks to find a predictive task assignment that minimizes the predicted parallel wall clock execution time $\hat{W}_{\text {par }}$.

Trivially, if $\hat{w}_{i} \neq w_{i}$ due to wrong predictions or cost indicators, then $\hat{W}_{p, M} \neq W_{p, M}$ for all $p \in P$ (except for some pathological cases). Thus, we may argue that any predictive load balancing is doomed to fail in an increasingly complex software and hardware environment.

Fig. 1 illustrates an example of how wrong predictions create imbalances. Here, a prediction model assumes uniform execution time for each task. If a rank slows down unexpectedly, idle times occur despite equal distribution of work.

\subsection{Reactive Load Balancing}

We can tackle the fundamental weaknesses of predictive load balancing in three different ways:

(1) Improve predictions: Improving the cost model and/or prediction function results in better predictions, hence in better task assignments. This may be achieved by adapting the cost model at run time (e.g., through performance instrumentation $[1,24]$ or auto-tuning [7]).

(2) Increase the frequency of load (re-)distribution steps and continuous performance monitoring: Traditional predictive load balancing approaches compute new task assignment mappings - followed by a load re-distribution step - in a certain interval after one or more task execution phases. Instead, new task assignment mappings and load distribution steps can be computed much more often during the task execution phase. This helps to iteratively improve load balance at run time by exploiting the most recent knowledge about the task execution progress. It reacts to the latest available performance data. This approach called reactive task migration was implemented in our previous work [15, 16, 23].

(3) Find a task assignment mapping reactively: Instead of relying on predictions in order to establish one (or multiple iteratively improved) task assignment mapping, a reactive strategy enables the task assignment mapping to adapt itself at run time through replication and cancellation of tasks. We focus on this approach in this work and explain more details in the following sections. All of the above strategies - if applied in isolation or in conjunction at run time during the task execution phase - enable load balancing to adapt to a changing execution environment. We call such load balancing algorithms reactive:

Definition 3.6 (Reactive Load Balancing Algorithm). Reactive load balancing adapts its task assignment $M$ and the respective task (re-)distribution during the task execution phase.

Reactivity of a load balancing approach is not a binary but rather a continuous property: depending on how frequently the task assignment can be changed and how often load can be re-distributed, load balancing may be considered more or less reactive. It is also not an exclusive property as load balancing can be predictive (use a cost model and/or prediction function) and reactive (adapt at run time) at the same time. Reactive task migration would be an example for a predictive and reactive load balancing scheme. Static load balancing algorithms are predictive per definition. Traditional dynamic load balancing algorithms are also mostly predictive. However, they can be considered as weakly reactive too, if they adapt at run time, e.g., by changing cost indicators or the prediction model $f$.

\section{REACTIVE REPLICATION-BASED LOAD BALANCING}

For simulation software that uses classic domain decomposition, data structures are either statically or dynamically distributed to processors. In most cases, the data distribution is chosen such that the respective work (i.e., the tasks for task-based applications) is balanced - although the distribution may be subject to other constraints, for example due to memory size. A data distribution establishes a unique mapping of computational work to a processor, as its required input data is only available on a single processor's main 
memory. For task-based parallel applications, tasks are therefore normally bound to their origin rank which can be defined as the process that spawns a task and "owns" its input data.

In our previous work $[15,16,23]$, the binding of tasks to a single process has been relaxed: tasks can be moved to a remote rank and be executed there. To maintain data consistency a task's output data is immediately sent back to the origin process. We follow this work and refer to this approach as task migration. In this section, we present extensions to support replication of tasks and their reactive execution for load balancing.

\subsection{Replication of Tasks between MPI Processes}

Tasks as an abstraction of data and respective work are natural candidates for replication and remote execution. They are called migratable tasks. Yet, not all tasks in an application may be suitable for execution on a different MPI process, for example due to the overhead of sending them to another rank [15, 16, 23]. We therefore suggested to migrate primarily compute-heavy tasks [16], whose processing time significantly exceeds the communication effort for migration. Migratable tasks are held in task queues for scheduling.

For replication, we plug into the spawning process of migratable tasks: whenever a migratable task $i$ is spawned by its origin rank $p_{\text {origin }}$, it is decided (based on some criteria that we detail later) whether this task shall be replicated on another rank. If not, the task is submitted to the task queue localTasks. Otherwise, the task is replicated to another replication victim rank $p_{\text {victim }}$ it becomes a replicated task on porigin, stored in the task queue replicatedTasks. This is detailed in Algorithm 1. A copy of a replicated task - a replica task - is migrated to $p_{\text {victim, where it is }}$ received and stored in a dedicated task queue replicaTasks.

Keeping replicated tasks and replica tasks in dedicated queues gives us more freedom for task scheduling, which we discuss later. Having replicated a task $i$, we end up with a situation where $i$ resides on both ranks, $p_{\text {origin }}$ and $p_{\text {victim. }}$. An example is illustrated in Fig. 2a and Fig. 2b.

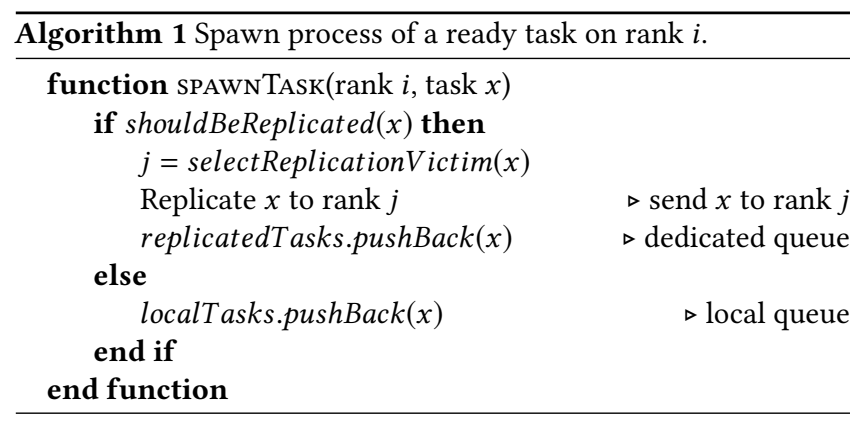

\subsection{Ensuring Data Consistency for Replicated Tasks}

In numerical software for parallel AMR, some data is persistently stored but iteratively updated throughout the simulation. Tasks that operate on parts of such persistent data are spawned repeatedly between iterations in iterative schemes. Keeping some data and the
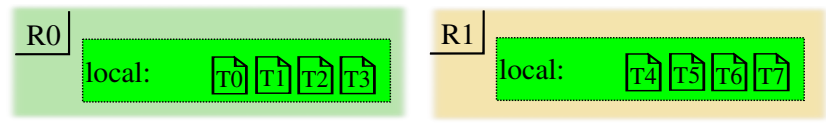

(a) No replication, all tasks are stored in the queue for local tasks.
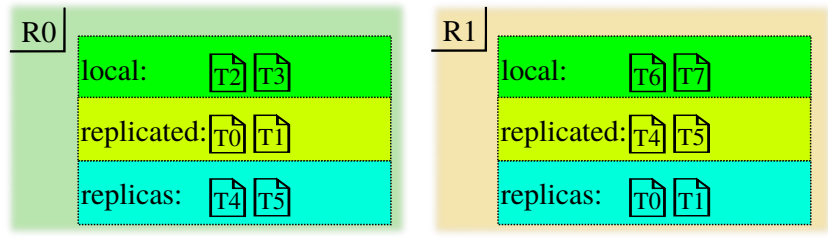

(b) Tasks 0, 1 are replicated onto rank 1. Tasks 4, 5 onto rank 0 .
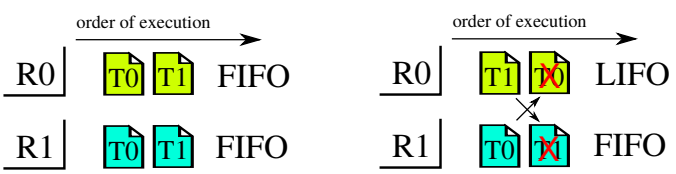

(c) FIFO (left, no cancellation possible) vs. LIFO (right, arrows and $x$ symbolize task cancellation) processing of replicated tasks. FIFO ordering of processing replicas results in redundant computation while LIFO processing allows for task cancellation, avoiding duplicate computation.

Figure 2: Example illustration of the content of task queues for a setup with two MPI ranks.

associated recurring tasks replicated leads to the problem of data consistency: if replicated tasks are not permanently executed on both $p_{\text {victim }}$ and $p_{\text {origin }}$, their associated output data would soon become inconsistent, requiring significant communication overhead and adding implementation effort for maintaining data consistency.

We follow a simpler approach: the logical ownership of a replicated task's output data belongs to the origin rank. Whenever a replicated task is sent to a $p_{\text {victim }}$ and processed there, its output data is immediately sent back and written into the origin rank's memory, i.e., the replicated data is held consistent on $p_{\text {origin. As }}$ soon as the output data transfer from $p_{\text {victim }}$ has finished, replica tasks and their data are deleted. Migrated tasks and their input and output data are never stored persistently on a remote victim rank. This automatically guarantees data consistency when tasks are replicated and avoids complex data consistency protocols.

\subsection{Reactive Execution of Replicated Tasks}

The semantics of task replication are speculative, i.e., if we replicate a task, we do not predictively decide whether it is executed on

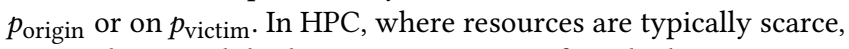
we need to avoid duplicate computations, for which we propose several reactive mechanisms.

Reactive Task Cancellation. Without specific measures, each replicated task would be computed twice on both $p_{\text {origin }}$ and $p_{\text {victim }}$. We therefore must be able to cancel a replicated task locally on $p_{\text {origin }}$ or its replica remotely on $p_{\text {victim. }}$. We never cancel tasks whose execution has already started (atomic tasks) and follow a simple first-come-first-served strategy instead: whichever rank delivers a 
replicated task's output first "wins" and tries to cancel its counterpart on the other rank (similar to [25]). Even with task cancellation, we may end up executing both a replicated task and its replica:

(1) Replicated task has finished execution locally, before a result from $p_{\text {victim }}$ is available: We send a cancel request to $p_{\text {victim. If the }}$ victim rank has already started processing the task before receiving the cancel request, we end up computing the task on both ranks. Otherwise, the task is canceled on $p_{\text {victim }}$ (remote task cancellation).

(2) Task output from $p_{\text {victim }}$ is available on $p_{\text {origin }}$ If $p_{\text {origin }}$ has not yet started the replicated task, the task is canceled (local task cancellation). Otherwise, $p_{\text {origin }}$ discards the result from $p_{\text {victim }}$ and finishes the local task execution.

Task cancellation happens during task execution. Algorithm 2 summarizes how execution and cancellation interact.

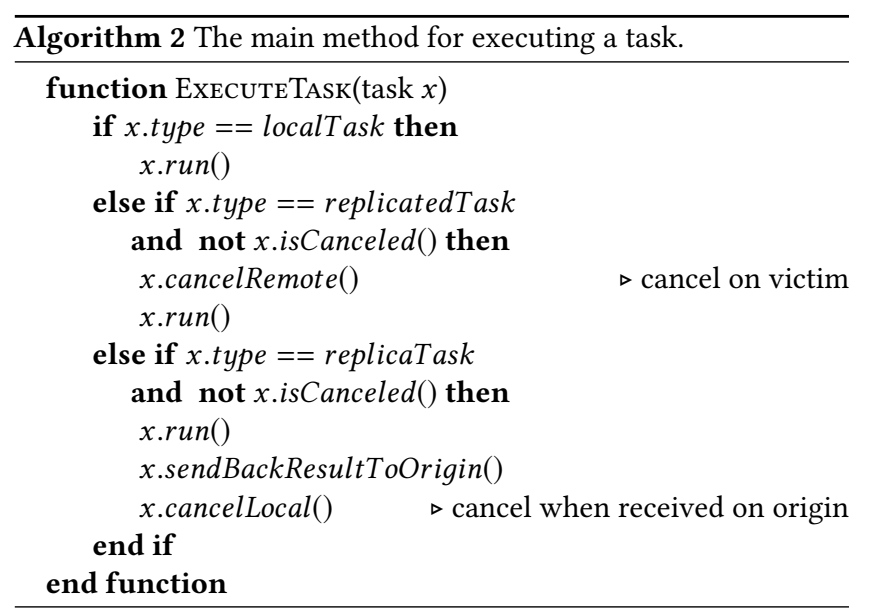

Reordering Replicated Tasks. We still need to decide on when replicated tasks and their replicas are scheduled for execution. In previous work, migrated tasks were scheduled with highest priority on $p_{\text {victim }}[15,16,23]$. This enables maximum overlap of communication and computation, as the result of migrated tasks is sent back as soon as possible. Proceeding in the same way for replicated tasks, however, would dismiss the purpose of replication which is to reactively decide at run time on whether a task is executed on $p_{\text {victim }}$ or on $p_{\text {origin. }}$ If we would process replicated tasks on $p_{\text {victim }}$ with high priority, they would always be canceled locally, i.e., we would bias the execution of replicated tasks toward $p_{\text {victim. }}$.

Instead, for a reactive approach, we adapt the task priorities for executing the three different types of tasks (local, replicated and replica tasks). Local tasks are scheduled with highest priority, i.e., before any replicated tasks or replica tasks are executed. Next, we schedule replicated tasks for execution: the reasoning is that we a-priori speculatively decide to replicate some tasks, but in a totally load balanced setup, these replicated tasks should be executed on their origin process. We therefore prioritize local execution over remote execution for a replicated task. Replica tasks are executed with the lowest priority - only if no more local work is available. but the task execution phase has not terminated globally. This way, rank $p_{\text {victim }}$ speculatively helps out $p_{\text {origin }}$.

Our approach effectively divides the task execution phase into three phases: processing of local tasks, processing of replicated tasks and then processing of replica tasks. Unpredictable load imbalances during a task execution phase always manifest in idle times at the end of the phase. By deferring replicated tasks to the end of the phase, we can reactively fill up remaining idle times on underloaded processes with replica tasks where most urgently needed. It is also at this point in the task execution phase, where other measures of proactively migrating tasks from overloaded to underloaded ranks would be less effective as there may not be many outstanding migratable tasks for redistributing work left anymore and communicating such tasks would induce additional latencies. Speculatively replicated tasks are already available at the time at which an imbalance is detected. Computing such replicated tasks can be seen as a last resort to react to load imbalance.

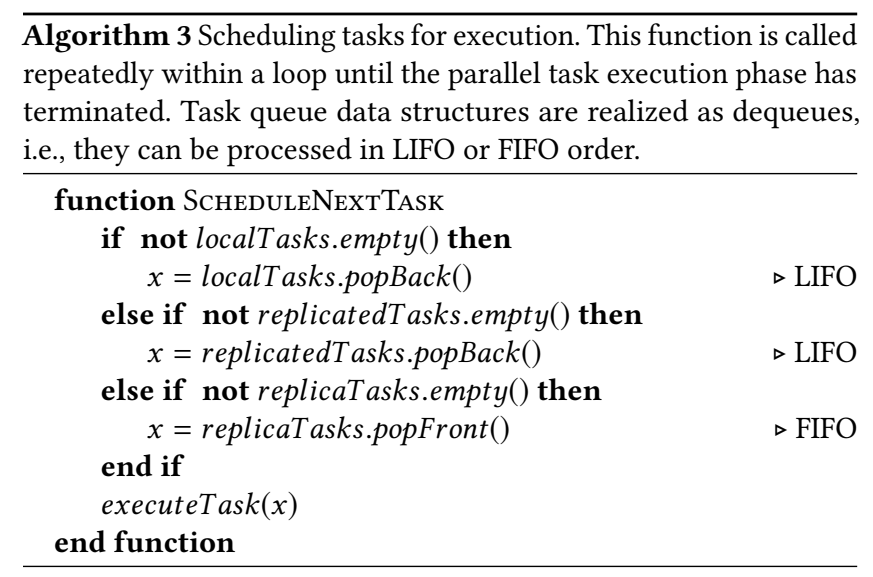

However, only delaying replicated tasks through prioritization is not sufficient to achieve optimal performance and reactive load balancing. Consider a scenario where replicated tasks are executed at the same time on $p_{\text {origin }}$ and $p_{\text {victim }}$, holding the corresponding replicas, both in FIFO order (same order in which they were created). If the executing ranks are perfectly synchronized, both replicated tasks as well as their replicas would be executed concurrently, leaving no opportunity for task cancellation. We would end up computing the replicated work twice for many replicated tasks. Therefore, we invert the processing order of replicated tasks to LIFO on their origin rank, while using FIFO processing for their replicas. In combination with task cancellation, $p_{\text {origin }}$ and $p_{\text {victim }}$ "move towards each other" when processing replicated work, i.e., they reactively determine an appropriate load balancing at run time. This also minimizes the risk of duplicated execution as illustrated in Fig. 2c. The main scheduling decisions are summarized in Algorithm 3.

\section{IMPLEMENTATION}

We implemented our reactive task replication strategies in the Chameleon library for reactive load balancing for hybrid MPI+OpenMP task-based parallel applications. Chameleon is publicly available ${ }^{1}$.

\subsection{Short Introduction to Chameleon}

5.1.1 Chameleon for Application Developers. Many parallel applications follow the bulk-synchronous programming approach of

\footnotetext{
${ }^{1}$ https://github.com/chameleon-hpc/chameleon
} 
Listing 1: Example code snippet that shows how Chameleon can be used in an application.

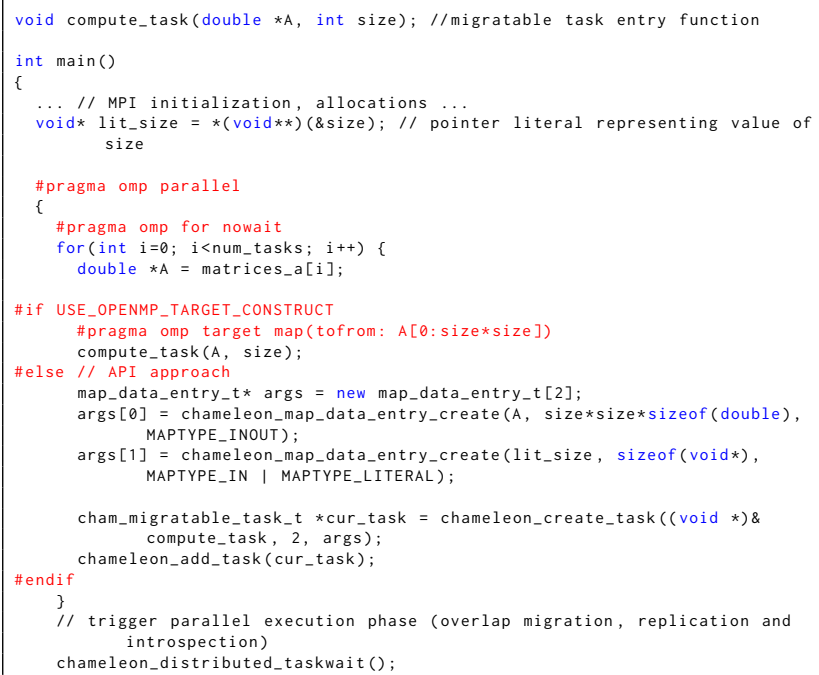

alternating computation and synchronization phases, where some communication may partially overlap with useful computation. For task-based MPI+OpenMP applications, the computation phases in bulk-synchronous programs can often be expressed in terms of compute-heavy tasks. These phases correspond to parallel task execution phases and are subject to Chameleon's load balancing. In Chameleon, tasks represents independent work packages without any side effects (e.g. access to global variables by more than one task) that can be executed in parallel, either on the local or on any other rank. Dependencies between tasks are not yet supported but are planned for future work.

Chameleon offers two different ways to expose tasks and their data environment as migratable Chameleon tasks to the library (see Lst. 1): (1) A pragma-based approach extends the OpenMP \#pragma omp target construct to support migratable tasks. This provides an elegant solution for developers to incrementally extend their application to support Chameleon's distributed tasking. The map clause is used to identify a task's input and output data. This approach was implemented for the Clang compiler by adding a custom libomptarget plugin $[15,16]$ for the LLVM OpenMP runtime. (2) For arbitrary $\mathrm{C}, \mathrm{C}++$ and Fortran compilers, Chameleon additionally provides a manual API for creating and adding Chameleon tasks and for providing information on their input and output arguments. More details on how to integrate Chameleon into applications and other code samples for both approaches can be found in our previous work [16].

Having created Chameleon tasks, the application passes on control to Chameleon for executing them on multiple MPI ranks using reactive load balancing. Throughout the task execution phase, the current load on each rank is continuously monitored and small messages holding each rank's load information are exchanged, providing a global view on the current load situation. Inspecting this data, Chameleon makes load balancing decisions, such as migrating tasks from overloaded to underloaded ranks. A migratable task and its data environment is serialized and transferred to another rank.

While Chameleon provides default strategies to interpret and define current load information and to react on them via migration decisions, the application programmer can affect or override these strategies by implementing callbacks of the Chameleon tools interface (similar to OMPT [11]). This interface allows an application to fully control Chameleon's decision making and to bring in domain-specific knowledge (e.g., on the predicted load of a task).

5.1.2 Chameleon Internals. Chameleon was designed keeping two key principles in mind: full overlap of load balancing, monitoring and related communication with the application execution and high reactivity. We therefore found it vital to dedicate one core in each MPI rank to an asynchronously running communication thread. The communication thread is responsible for repeatedly exchanging current load information between ranks, making task migration decisions, and for progressing any outstanding non-blocking MPI communication of migrated tasks or their output (similar to [4, 14, 27]). Exchanging current load information repeatedly also allows us to piggyback a simple termination criterion: whenever all tasks in a parallel task execution phase have finished, control is given back to the application. All this happens in the background, while the application and its computation threads are processing migratable (and possibly migrated) tasks.

\subsection{Replication of Tasks in Chameleon}

5.2.1 Implementation of Task Cancellation. Implementing task cancellation in Chameleon is delicate as several steps are involved that can be executed in any order in a multi-threaded environment: task execution on the origin rank, task execution on the victim rank, receiving a task's output and writing it into the origin's main memory and processing cancel requests need to be orchestrated. Particularly, data consistency must be maintained, i.e., processing a replicated task locally and receiving the result from the replication victim should not happen in parallel to avoid conflicting concurrent memory accesses. Moreover, a remote task cancellation request may arrive before or after a replica task has started.

We coordinate the data consistency issue by assigning the responsibility for executing a task and writing its output data to a single thread. Synchronization is achieved by using thread-safe task queues where both the communication as well as one of the computation threads compete for a replicated task: whenever task output data from a replica task is available on the communication thread, it tries to remove the task from the thread-safe task queue before it is allowed to write the task's result. If successful, the task was canceled locally.

On the other hand, if a computation thread has dequeued the task before the task output data is available from a victim rank, the computation thread executes the task and writes the result. If the communication thread receives the task output after a computation thread has started to compute the task, it ignores the output data by receiving it into a "trash buffer".

For remote task cancellation, we equip each replicated task with its replication victim ranks (stored in the task data structure). If an origin rank decides to compute a replicated task locally, it sends the 
task's unique id to all replicating victim ranks. Upon receiving such a cancellation request, the victim rank will try to remove the replica task from its task queue. Depending on whether the victim rank has already started processing the task or not, the task has been canceled successfully, corresponding to remote task cancellation.

5.2.2 Selecting Tasks for Replication and Choosing Victim Ranks. Algorithm 1 needs to decide whether a task should be replicated and if so, which other MPI ranks receive a replica copy of this task. The replication strategy can be customized by the application through the Chameleon tools interface. In this work, we use the following default replication strategy: To limit the communication overhead caused by replication, we replicate tasks to at most one other rank, although we support replication factors $>2$. For selecting tasks to replicate and replication victims, we combine two strategies: speculative a-priori replication and replication of migrated tasks.

Speculative a-priori replication selects the first $r_{\max }$ of all spawned tasks in a task execution phase for replication which can be limited by the user through an environment variable. This is to be read weakly, as depending on factors such as the task granularity, the task processing rate and the available communication bandwidth, Chameleon may only replicate a smaller number $x<r_{\max }$ of all spawned tasks. For choosing replication victims, we do not want to make any predictions about which rank may be overloaded or not. Fully speculatively, we replicate $x / 2$ tasks of a rank $p$ to the neighboring rank $p-1$ and the other $x / 2$ tasks to rank $p+1$ (modulo the total number of ranks). This a-priori replication creates some buffer for reactive load balancing in urgent cases.

Chameleon also closely monitors current load data and makes respective task migration decisions in a predictive way. A migrated task - in contrast to a replicated task - is deterministically executed on its remote victim rank. However, Chameleon can be configured such that migrated tasks are automatically also kept on their origin rank $p_{\text {origin. }}$. This way, a migrated task $i$ automatically becomes a replicated task, subject to reactive execution of replicated tasks. Local execution of $i$ and remote task cancellation then can mitigate wrong migration decisions, which we demonstrate in Sec.6.

\section{EVALUATION}

$\mathrm{Sam}(\mathrm{oa})^{2}$ is a framework for solving PDE systems on dynamically adaptive tree-structured triangular meshes [17]. For shared- and distributed-memory parallelism, $\operatorname{sam}(\mathrm{oa})^{2}$ sub-partitions the mesh into sections of grid cells that are contiguous along a Sierpinski space-filling-curve. These "Sierpinski sections" act as atomic units for load balancing and also as tasks for OpenMP parallelization. Predictive chains-on-chains partitioning (CCP) [19] with the number of cells as a cost indicator of a section is used for domain decomposition. Sam $(\mathrm{oa})^{2}$ was extended to support distributed reactive tasking with the Chameleon library on top of CCP load balancing. In our tests, we spawn 16 tasks per thread per time step on each MPI rank, yielding a sufficient degree of over-decomposition.

For the following tests, we consider models for solving the $2 D$ shallow water equations applied for tsunami simulations as use-cases. $\operatorname{Sam}(\mathrm{oa})^{2}$ provides both a finite volume solver (with regularly-refined grid patches for better vectorization $[12,17])$ as well as an implementation of a high-order discontinuous Galerkin scheme (ADER-DG, [20]) with a-posteriori limiting. ADER-DG in sam $(\mathrm{oa})^{2}$ can be decomposed into three phases which are subject to Chameleon's load balancing: (1) A space-time predictor (STP) approximates an element-local solution by means of a fixpoint iteration loop. The number of iterations is not known a-priori and can cause load imbalances. (2) Riemann problems are solved at the element boundaries using the STP solution (which is extrapolated to the boundaries). (3) A corrector step updates the STP solution by taking into account the solution to the Riemann problems. Further, a limiter identifies elements where the solution violates physical admissibility criteria and recomputes it in these cells on a finer finite volumes patch in the corrector step (cf. [10]). Variations in computational cost per element are caused by the limiting procedure and by the space-time predictor in ADER-DG, but also by the numerical treatment of inundation at coast lines. We spawn a section traversal in the time stepping phase (ADER-DG + Finite Volumes) as well as for computing the STP (ADER-DG only) as a Chameleon task, i.e., these tasks can possibly be executed on different MPI ranks.

We ran tests on SuperMUC-NG, hosted by the Leibniz Supercomputing Centre ${ }^{2}$. SuperMUC-NG features Intel Skylake Xeon Platinum 8174 dual-socket compute nodes with 48 cores (clocked at a nominal frequency of $2.3 \mathrm{GHz}$ ) and 96 GByte of main memory. Nodes are connected in a fat tree topology based on Intel OmniPath.

We evaluated several variants of running the benchmarks: In the baseline, $\operatorname{sam}(\mathrm{oa})^{2}$ does not use Chameleon to execute tasks remotely or to replicate them. The reactive task migration variant does not keep tasks replicated but it may select some tasks and migrate them to a different MPI rank at run time if load imbalances are detected using a prediction for a rank's current load (cf. [16]). In the third variant reactive task migration + replication, migrated tasks are replicated (i.e., a local copy is kept on the origin rank) and apriori replication is active, i.e., Chameleon decides to speculatively replicate as many tasks as possible between neighboring ranks (cf. Sec. 5).

\subsection{Overhead of Replication and Task Migration}

As reactive task migration and replication add communication work, we first examined the overhead. We use a static uniform grid with $4.11 \cdot 10^{8}$ cells to simulate 1000 time steps of the perfectly workbalanced radial dam break scenario with the finite volume solver. We scale this setup up to 512 compute nodes of SuperMUC-NG (strong scaling). As the work is perfectly load balanced and there is no noise, there is generally no need for any reactive load balancing. Therefore, this test setup also qualifies as a robustness check: reactive task migration and replication should not significantly interfere with the baseline performance.

Our measurements in Fig. 3 show that up to 128 nodes (256 ranks), all three variants scale up with a parallel efficiency $\geq 0.8$. No significant performance impact of using Chameleon on the time-to-solution is noticeable for these setups where the parallel efficiency is acceptable. The reactive tasking mechanisms only add minor overhead, mainly for a-priori replication of tasks and task cancellation. It is only beyond the strong scaling limit (parallel efficiency $<0.8$ ) where the communication overhead becomes slightly visible (cf. the data with 1024 ranks). In this case, there is little

\footnotetext{
${ }^{2}$ https://doku.lrz.de/display/PUBLIC/SuperMUC-NG
} 


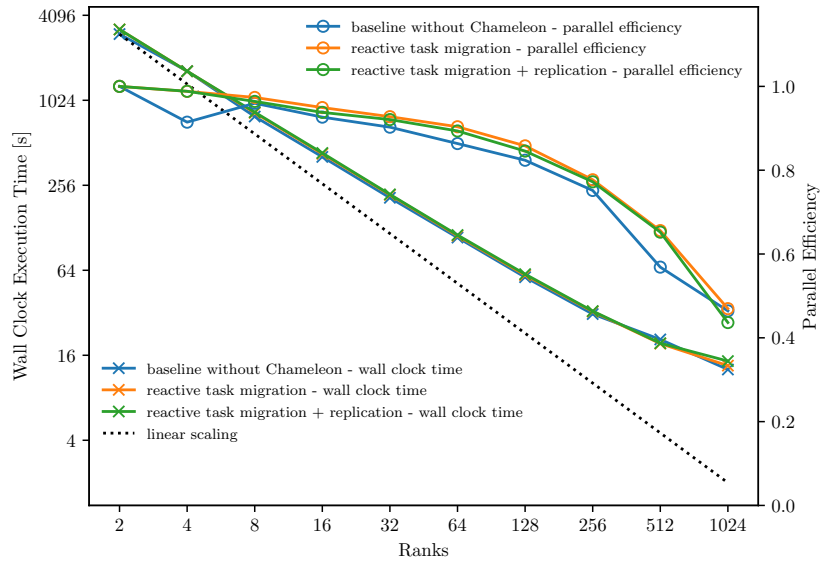

Figure 3: Wall clock time and parallel strong scaling efficiency on up to 512 nodes of the following variants of running the radial dam break scenario with $\operatorname{sam}(\mathrm{oa})^{2}$ : baseline without Chameleon (24 OpenMP threads, 1 rank per socket), reactive task replication and migration (23 OpenMP threads + 1 Chameleon thread, 1 rank per socket).

potential to overlap the overhead (e.g. for canceling replicated tasks) with useful computation. Inspecting our output logs for 1024 ranks more closely, we found a very small fraction of about $0.1 \%$ duplicate executions (relative to all created tasks): the task cancellation did not fully keep up with the task processing rate. Yet, in this test case, we may argue that we obtain replication of tasks without most of the cost of duplicate computations. Comparing the baseline with 24 cores per rank with the reactive Chameleon variants, we can observe that losing one core for the communication thread in Chameleon does not significantly degrade performance (see also [16, 23]): all three variants of sam(oa $)^{2}$ perform similarly.

\subsection{Robustness against Misleading Predictions}

Predictive load balancing can only be as good as its predictions. We expect that in the future it will become increasingly difficult to come up with accurate load predictions. In this strong scaling experiment, we investigate the robustness of our reactive load balancing approaches under an artificially disturbed prediction model. We simulate 1000 time steps of the balanced radial dam break scenario with a uniform grid of $4.11 \cdot 10^{8}$ cells. We, however, artificially provoke wrong predictions for the reactive task migration component in Chameleon as well as for the CCP load balancing by actively disturbing the cost indicator for the work of the tasks on odd ranks leading to mis-predictions of the actual computational work: odd ranks are believed to only have half of the workload which they actually have. Thus, we are creating a reproducible worst-case scenario to evaluate the capabilities of our solution that induces a load imbalance, as odd ranks get assigned more work.

The results in Fig. 4 show the speedups/slowdowns of the reactive load balancing variants with respect to the baseline, where we scale up the number of ranks. We also plot the measured load imbalance (an imbalance of 1.0 means perfectly balanced) for the different runs. With a disturbed prediction model, sam(oa) ${ }^{2}$ only

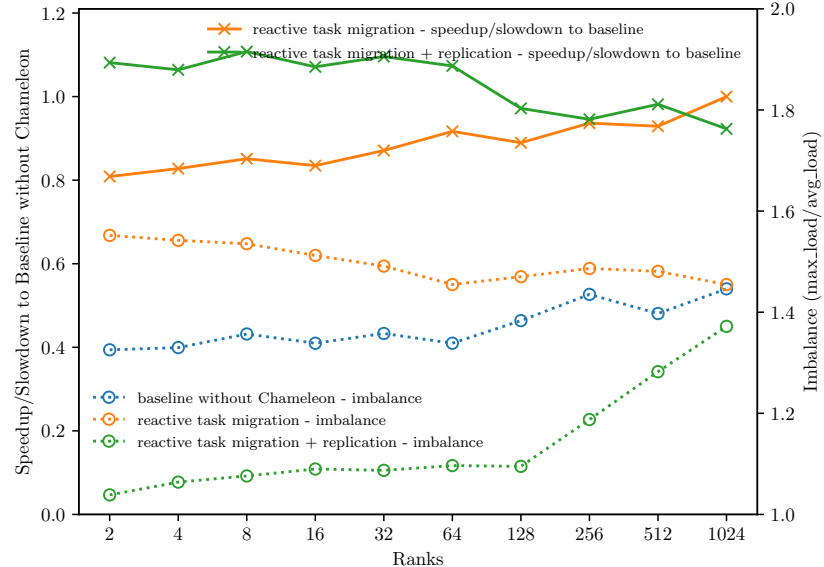

Figure 4: Speedups/slowdowns to baseline and measured load imbalance without Chameleon (24 OpenMP threads per rank), with reactive task migration and replication (23 OpenMP threads +1 Chameleon thread) in the presence of wrong predictions at different numbers of ranks on up to 512 nodes (1 rank per socket, radial dam break scenario, SuperMUC-NG).

reaches suboptimal load balance. Even reactive task migration is still weakly predictive, as it bases its load balancing decisions on estimated load. As soon as its load information is disturbed, reactive task migration actually increases load imbalance compared to the baseline by overloading some ranks through misleading task migration decisions. It is important to note that this effect is a fundamental weakness of any predictive load balancing algorithm.

In contrast, adding replication of migrated tasks on top of reactive task migration achieves an almost balanced application execution, outperforming both the baseline as well as the variant that uses only task migration: as migrated tasks are also replicated in this setup (i.e., they are still available for local execution on the origin rank) any wrong predictive migration decisions are undone by computing wrongly migrated tasks locally and canceling their replica copies remotely. On top, a-priori replicated tasks help to better overlap communication and computation and thus further reduce the work imbalances between different MPI ranks. We may conclude that keeping tasks replicated and delaying their scheduling to the end of the task execution phase can help increase robustness against wrong predictions for a predictive load balancing approach.

As we reach the strong scaling limits, the above effects become less pronounced: as expected, the relative costs of task migration and replication increase such that Chameleon decides to reduce its communication overhead by migrating less tasks. Yet, we do not obtain significant performance penalties relative to the baseline.

\subsection{Handling Unpredictability in the ADER-DG Scheme}

Next, we examine how the different reactive load balancing strategies in Chameleon perform in the presence of unpredictable task 
execution costs. We use the ADER-DG variant of sam(oa $)^{2}$ to simulate 100 time steps of the realistic oscillating lake scenario [3] with a uniform mesh on 16 and 32 nodes (strong scaling) on SuperMUCNG. Here, future load is highly unpredictable, due to wetting and drying and due to the limiter being dynamically applied to some cells of a section. We do not falsify any cost indicators for determining a task's load: imbalances are merely caused by the numerics.

Tab. 1 shows the performance data for different configurations of reactive load balancing in Chameleon. We varied the choice of replication and migration mode: the first column in Tab. 1 determines whether Chameleon may decide to migrate tasks to other ranks during the task execution phase (based on load monitoring). A 'yes' in the second column indicates that migrated tasks are also replicated, i.e., a local copy is kept on the origin rank. The third column determines whether Chameleon also speculatively replicates tasks. We report measurements for runs at 24 threads per rank: together with the Chameleon communication thread, the full socket is always used. The baseline (first row, ran with 24 OpenMP threads) is only using the CCP predictive load balancing to evenly decompose the uniform grid, distributing the grid uniformly across the nodes. Reactive load balancing with Chameleon is disabled.

The baseline only shows suboptimal performance when compared to the variants with some reactive load balancing enabled. Speculative replication has a small negative impact on throughput. Best performance is obtained when reactive task migration is active and speculative replication is inactive.

Speculative replication comes at some cost: it adds communication overhead for replicating tasks to the neighboring ranks without guaranteeing that the replication victims will actually be able to process replicated tasks and thus, to help out the origin ranks effectively. In fact, we noticed when using only speculative replication with our employed default replication strategy (replicate between consecutive ranks), it was not possible to balance load evenly due to clusters of consecutive overloaded ranks. Moreover, with speculative replication, the communication pressure of canceling tasks for avoiding duplicate computations increases, especially at high thread counts. Finally, in our implementation, replicated tasks can no longer be migrated, limiting the number of tasks available to the reactive task migration mechanism.

We obtain speedups of about $2.91 \times$ for 16 nodes and of about $2.64 \times$ for 32 nodes. The combination of task migration with replication of migrated tasks seems to be an attractive choice for this scenario: keeping migrated tasks replicated comes at hardly any additional expense but could help to tackle misleading predictions as shown in the previous section. In contrast to merely speculative replication, reactive load monitoring ensures that communication resources are used to make effective migration decisions.

\subsection{Pushing Predictive Load Balancing to the Limits}

Eventually, future exascale systems may be so unpredictable such that even the reactive task migration may not only mis-predict a task's load but it may also be inactive in detecting load imbalances and in reacting accordingly. Therefore, we push predictive load balancing to such absolute limits, by means of an extreme (but controlled) test environment.
Table 1: Performance for the Oscillating Lake scenario on a uniform grid with about $9 \cdot 10^{8}$ degrees of freedom on 16 and on 32 nodes. We vary the choice of replication and migration mode, indicated by 'yes' if a feature is used and 'no' otherwise.

\begin{tabular}{rrr|rc} 
& & & \multicolumn{3}{|c}{ Total million cell updates/s } \\
Task mi- & Rep. of & A-priori & 24 threads & 24 threads \\
gration & mi- & specula- & per rank, & per rank, \\
grated & tive & 16 nodes & 32 nodes \\
tasks & rep. & & \\
\hline no & no & no & 105.63 & 204.63 \\
yes & no & no & 307.92 & 540.50 \\
yes & yes & no & 270.05 & 528.90 \\
yes & yes & yes & 245.29 & 530.85 \\
no & no & yes & 123.46 & 205.42
\end{tabular}

On each even rank, we add noise to the tasks at run time. As it was technically not possible to change the CPU clock frequencies on our machine, we inserted sleeping cycles into the task execution for simulating noise. The noise sets in after half of the tasks on a disturbed rank have been processed in a parallel task execution phase. On top, we disturb the cost indicator for the work of the tasks such that the total load on disturbed ranks is underestimated by Chameleon. In summary, we mimic a setup where multiple nodes suddenly clock down during task execution and where the load information is disturbed such that these events cannot be predicted.

We simulate 1 hour of the Indian Ocean tsunami generated by the 2004 Sumatra-Andaman earthquake [20]. We use a dynamically adaptive mesh on 16 nodes on SuperMUC-NG with the ADER-DG solver at order 4 . As the production-level simulation runs took several hours of wall-clock time until completion, we only report single run measurements. In Tab. 2, we again report the performance data for different configurations of running our benchmark.

Unpredictability of performance in a noisy runtime environment makes it particularly challenging to achieve good load balance and hence good performance. With reactive task migration, imbalances in the baseline without any reactive load balancing (first row in Tab. 2) are reduced greatly, resulting in a performance speedup of up to $1.51 \times$. Yet, predictive performance data still remains uncertain: reactive task migration may make wrong migration decisions (cf. 6.2) or remain inactive. Therefore, best performance is achieved only when tasks are replicated speculatively but no (possibly misleading) predictive load balancing decisions are made leading to a speedup of $1.74 \times$. It is exactly in this regime of highly uncertain performance predictions where a-priori replication and reactive execution of replicated tasks provides the best remedy to mitigate load imbalance. This implies that only if all predictive mechanisms fail, a-priori replication without migration provides the best choice. In most other cases, a combination of reactive task migration and replication seems most attractive (cf. 6.3).

\section{CONCLUSION}

We presented a novel approach for task-based load balancing in distributed memory, which uses speculative replication of tasks to other MPI processes and their reactive execution using task 
Table 2: Performance for the Sumatra scenario with dynamic AMR with about $4.5 \cdot 10^{6}$ degrees of freedom on average on 16 nodes under artificial noise conditions. We vary the choice of replication and migration mode, indicated by 'yes' if a feature is used and 'no' otherwise.

\begin{tabular}{rrr|r}
$\begin{array}{r}\text { Task mi- } \\
\text { gration }\end{array}$ & $\begin{array}{r}\text { Replication } \\
\text { of migrated } \\
\text { tasks }\end{array}$ & $\begin{array}{r}\text { A-priori } \\
\text { speculative } \\
\text { replication }\end{array}$ & $\begin{array}{r}\text { Total million } \\
\text { cell updates/s } \\
(24 \text { threads per } \\
\text { rank })\end{array}$ \\
\hline no & no & no & 88.58 \\
yes & no & no & 136.93 \\
yes & yes & no & 135.27 \\
yes & yes & yes & 139.48 \\
no & no & yes & 154.57
\end{tabular}

cancellation. In contrast to traditional load balancing, our method is fully reactive as it does not rely on any performance predictions. We implemented this approach in the Chameleon library for reactive load balancing and evaluated it with the sam(oa $)^{2}$ framework for dynamically parallel AMR. Due to its fully overlapping nature, the method introduces only little overhead. One of its biggest strengths is that it provides new reactive mechanisms for achieving load balance in situations in which other predictive measures would fail.

We see this work as a fundamental (yet still prototypical) contribution to future parallel computing. While predictive load balancing still works well for many applications, our migration and replication techniques provide an add-on to classic predictive load balancing methods that prepares task-parallel applications for the increasing unpredictability of execution time for new numerical algorithms on future systems.

\section{ACKNOWLEDGMENT}

This work was funded by the German Federal Ministry of Education and Research (BMBF) as project "Chameleon" (project no. 01IH16004). Compute resources were provided by the Leibniz Supercomputing Centre and by JARA-HPC from RWTH Aachen University under projects jara0001 and supp0001. We especially thank Leonhard Rannabauer for his extensive support on the ADER-DG code in sam(oa $)^{2}$. Thanks are also due to Karl Fürlinger and Christian Terboven for their valuable feedback and suggestions.

\section{REFERENCES}

[1] B. Acun, A. Gupta, N. Jain, A. Langer, H. Menon, E. Mikida, X. Ni, M. Robson, Y. Sun, E. Totoni, L. Wesolowski, and L. Kale. 2014. Parallel Programming with Migratable Objects: Charm++ in Practice. In Proc. Int. Conf. High Performance Computing, Networking, Storage and Analysis (SC '14). IEEE Press, 647-658.

[2] B. Acun, P. Miller, and L. V. Kale. 2016. Variation Among Processors Under Turbo Boost in HPC Systems. In Proc. 2016 Int. Conf. Supercomputing (Istanbul, Turkey) (ICS '16). ACM, New York, NY, USA, Article 6, 12 pages.

[3] Emmanuel Audusse, François Bouchut, Marie-Odile Bristeau, Rupert Klein, and Benoit Perthame. 2004. A Fast and Stable Well-Balanced Scheme with Hydrostatic Reconstruction for Shallow Water Flows. SIAM J. Sci. Comput. 25 (01 2004).

[4] D. Buettner, J.-T. Acquaviva, and J. Weidendorfer. 2013. Real Asynchronous MPI Communication in Hybrid Codes Through OpenMP Communication Tasks. In Proc. 2013 Int. Conf. Parallel \& Distributed Systems (ICPADS '13). IEEE Computer Society, 208-215.

[5] C. Burstedde, L. C. Wilcox, and O. Ghattas. 2011. p4est: Scalable Algorithms for Parallel Adaptive Mesh Refinement on Forests of Octrees. SIAM 7. Sci. Comput. 33, 3 (2011), 1103-1133.
[6] J. Charles, P. Jassi, N. S. Ananth, A. Sadat, and A. Fedorova. 2009. Evaluation of the Intel Core i7 Turbo Boost feature. In 2009 IEEE Int. Symp. Workload Characterization (IISWC). 188-197.

[7] D. E. Charrier and T. Weinzierl. 2018. An experience report on (Auto-)tuning of mesh-based PDE solvers on shared memory systems. In Lecture Notes in Computer Science, Vol. 10778. 3-13.

[8] J. Dean and S. Ghemawat. 2008. MapReduce: Simplified Data Processing on Large Clusters. Commun. ACM 51, 1 (Jan. 2008), 107-113.

[9] A. Dubey, A. C. Calder, C. Daley, R. T. Fisher, C. Graziani, G. C. Jordan, D. Q. Lamb, L. B. Reid, D. M. Townsley, and K. Weide. 2013. Pragmatic optimizations for better scientific utilization of large supercomputers. Int. f. High Perform. Comput. Appl. 27, 3 (2013), 360-373.

[10] M. Dumbser and R. Loubère. 2016. A simple robust and accurate a posteriori subcell finite volume limiter for the discontinuous Galerkin method on unstructured meshes. f. Comp. Phys. 319 (2016), 163-199.

[11] A. E. Eichenberger, J. Mellor-Crummey, M. Schulz, M. Wong, N. Copty, R. Dietrich, X. Liu, E. Loh, and D. Lorenz. 2013. OMPT: An OpenMP tools application programming interface for performance analysis. In Lecture Notes in Computer Science, Vol. 8122. 171-185.

[12] C. R. Ferreira and M. Bader. 2017. Load Balancing and Patch-Based Parallel Adaptive Mesh Refinement for Tsunami Simulation on Heterogeneous Platforms Using Xeon Phi Coprocessors. In PASC'17 - Proc. Platform for Adv. Scient. Comp. Conf. ACM.

[13] M. Garcia, J. Corbalan, and J. Labarta. 2009. LeWI: A Runtime Balancing Algorithm for Nested Parallelism. In 2009 Int. Conf. Parallel Processing. 526-533.

[14] T. Hoefler and A. Lumsdaine. 2008. Message progression in parallel computing-to thread or not to thread? In 2008 IEEE Int. Conf. Cluster Computing. 213-222.

[15] J. Klinkenberg, P. Samfass, M. Bader, and C. Terboven. 2020. Reactive Task Migration for Hybrid MPI+OpenMP Applications. In Parallel Processing and Applied Mathematics. Springer, 59-71.

[16] J. Klinkenberg, P. Samfass, M. Bader, C. Terboven, and M. S. Müller. 2020. CHAMELEON: Reactive Load Balancing for Hybrid MPI+OpenMP Task-Parallel Applications. F. Parallel Distr. Comp. 138 (2020), 55-64.

[17] O. Meister, K. Rahnema, and M. Bader. 2016. Parallel Memory-Efficient Adaptive Mesh Refinement on Structured Triangular Meshes with Billions of Grid Cells. ACM Trans. Math. Softw. 43, 3 (2016), 1-27.

[18] OpenMP Architecture Review Board. 2018. OpenMP Application Program Interface, Version 5.0. http://www.openmp.org/.

[19] A. Pinar and C. Aykanat. 2004. Fast optimal load balancing algorithms for 1D partitioning. 7. Parallel Dist. Comp. 64, 8 (2004), 974-996.

[20] L. Rannabauer, M. Dumbser, and M. Bader. 2018. ADER-DG with a-posteriori Finite-Volume limiting to simulate tsunamis in a parallel adaptive mesh refinement framework. Comp. Fluids 173 (2018), 299-306.

[21] A. Reinarz, D. Charrier, M. Bader, L. Bovard, M. Dumbser, K. Duru, F. Fambri, A-A. Gabriel, J-M. Gallard, S. Köppel, L. Krenz, L. Rannabauer, L. Rezzolla, P. Samfass, M. Tavelli, and T. Weinzierl. 2020. ExaHyPE: An Engine for Parallel Dynamically Adaptive Simulations of Wave Problems. Comp. Phys. Comm. 107251 (2020).

[22] J. Reinders. 2007. Intel Threading Building Blocks (first ed.). O’Reilly \& Associates, Inc.

[23] P. Samfass, J. Klinkenberg, and M. Bader. 2018. Hybrid MPI+OpenMP Reactive Work Stealing in Distributed Memory in the PDE Framework sam(oa) ${ }^{2}$. In 2018 IEEE Int. Conf. Cluster Computing (CLUSTER). 337-347.

[24] P. Samfaß, T. Weinzierl, D. E. Charrier, and M. Bader. 2020. Lightweight task offloading exploiting MPI wait times for parallel adaptive mesh refinement. Concurr. Comput. (2020).

[25] P. Samfass, T. Weinzierl, B. Hazelwood, and M. Bader. 2020. teaMPI-replicationbased resiliency without the (performance) pain. In High Performance Computing, 35th Int. Conf. ISC High Performance 2020, Vol. 12151. 455-473.

[26] T. Weinzierl. 2019. The Peano Software - Parallel, Automaton-based, Dynamically Adaptive Grid Traversals. ACM Trans. Math. Softw. 45, 2 (2019), 14:1-14:41.

[27] M. Wittmann, G. Hager, T. Zeiser, and G. Wellein. 2013. Asynchronous MPI for the Masses. CoRR abs/1302.4280 (2013). arXiv:1302.4280

[28] O. Zanotti, F. Fambri, M. Dumbser, and A. Hidalgo. 2015. Space-time adaptive ADER discontinuous Galerkin finite element schemes with a posteriori sub-cell finite volume limiting. Comp. Fluids 118 (2015), 204-224. 